\title{
Close Relation between Prothrombin Time and Thiamin; Contribution to the Study of Normal Prothrombin Time. Preliminary Report.
}

\author{
By
}
Akira Sato, Kakujiro Sakai and Katsumi Hayashi.
(佐 藤彰) (堺 鶴二郎)
(林 克 已)

(From the Department of Pediatrics, Faculty of Medicine, Tohoku

University, Sendai. Director: Prof. A. S a to.)

(Received for publication, May 13, 1949)

The close relation between vitamin $\mathrm{K}$ deficiency and hypoprothrombinemia is well known. But as to the intimate relation between prothrombin time and thiamin, there has been no report within our knowledge. Here we shall report preliminarily on this relation.

It was rather accidental that we could notice the alleged intimate relation. We had been investigating prothrombin time of some diseased children, but we had used healthy lactating mothers as control, irrespective

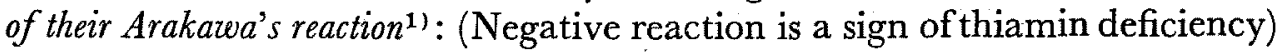
-because we had thought that even Arakawa-negative mothers, apparently healthy, could serve as controls as well. In the course of investigation, however, suspicion was aroused that prothrombin time might possibly be prolonged in them. Hence a systematic investigation into the relation between prothrombin time and Arakawa's reaction, or into the relation between prothrombin time and thiamin deficiency.

We studied it in 270 cases of lactants ${ }^{2}$ and found that Arakawa-negative mothers-lactants with thiamin deficiency-had a more or less prolonged prothrombin time, further that thiamin supply to such cases brought it down to the normal. On the other hand we examined 200 cases of breast-fed infants nursed with milk of different Arakawa's reaction and found that those nursed with Arakawa-negative milk showed a more or less prolonged prothrombin time. Further we found that thiamin administration brought it down to the normal. It was not rare that prothrombin time, apparently normal, was shortened by 4 or 5 seconds by thiamin injection.

Thus, women in labour ought not be suffering from vitamin $K$ deficiency, but also from thiamin deficiency either.

\section{Literature.}

1) Cf. many Reports on the Peroxidase Reaction published in this Joumal since 1930. 2) K. Sakai, Tohoku J. Exp. Med., (220th and 221 st Reports of the Peroxidase Reaction) to be published in a fourthcoming issue. 3) K. Hayashi, Ibid. (243rd and 245th Reports on the Peroxidase Reaction) to be published in a forthcoming issue. 